\title{
Introduction: Social Ontology, Culture and Institutions
}

\author{
Alessandro Salice $^{1} \cdot$ Filip Buekens ${ }^{2}$
}

Published online: 28 December 2015

(C) Springer Science+Business Media Dordrecht 2015

In this issue, six authors discuss, from various perspectives, the interaction between culture, evolution and institutions. Kim Sterelny's topic is language evolution, with its challenge being to develop an incremental model of change that takes us by small improvements from the baseline communicative capacities of great apes to those of contemporary humans, which, Sterelny argues with Jackendoff, are characterized by expressive richness, i.e. the capacity to freely innovate at the semantic level. A theory about language evolution must be nested within an overall conception of the evolution of social life (Tomasello 2008). Communication is one form of cooperation, and the evolution of social life is characterized by the expanding role of the economic basis of cooperation. Sterelny argues that, at a first stage, signals are meaningful without users' being aware that they are meaningful (Skyrms 2010). Signals, on the Lewis-Skyrms model, depend on asymmetries between signaler and receiver: one has access to information that the other lacks, and there must be an overlap of interests between the agents. As the recognition-signal-reception-action loop can be reflex-like, we must ask how we get from a reflex-like communication system to human language, with its key characteristic being that communication is flexible and brought under intentional control. Iconicity and association have a limited domain of potential signals. Once the basic flow of communication depends on arbitrary signals, it is harder to see how the expressive powers of a community's communicative practices could expand by happy accident in innovation and response. Once gesture has become conventionalized, or

Filip Buekens

Filip.Buekens@hiw.kuleuven.be

1 University of Copenhagen, Copenhagen, Denmark

2 Tilburg University and KU Leuven, Louvain, Belgium replaced by arbitrary vocal signals, intention and interpretation seem to be essential. Signal uptake can no longer be mediated by associative links between the signal and the action or actor the signal labels. Sterelny holds that, once the mime-gesture system has begun to expand, and the menu of signals expands beyond those with associative links to functional, non-communicative actions, the uptake and spread of innovation in expressive power will depend mostly on intelligent innovation and response. The sign sequence must be successfully exploited by the audience, to allow the innovator and the audience to coordinate in action. Sterelny's paper finishes with a summary of empirical findings that link the changes in signaling capacities with technical, economic and social revolutions between 500,000 and 80,000 years ago.

Ramsey and De Block discuss a central divide between those approaches to culture that center on replicators (or memes) and those that center on organisms, and they provide an extensive argument for the superiority of the organism-centered view over the meme-centered view of cultural evolution. According to Dawkins and other memeticists, cultural traits spread not because they are useful for the individuals with these traits but, mainly (and often even solely), because they aid meme propagation. For those who favor the meme's approach, the prototypical meme is the chain letter: a chain letter promises punishment for those who don't distribute it further (or reward for those who do) and, hence, influences events such that it gets replicated over and over. The organism approach, on the other hand, takes the organisms and the cultural traits they adopt to be the locus of cultural evolutionary theory. It is concerned with understanding the spread and impact of cultural traits, but radically differs with respect to the question of what counts as an instance of replication. Replication, according to the approach Ramsey and De 
Block favor, occurs when a naïve individual adopts a cultural variant whereas, for the memetic approach, replication can occur in the absence of organisms' adopting the variant.

Note that Ramsey and De Block do not argue that the organism-based approach (OC) is always superior, or that it is the only model of culture that one will ever need, but that, for the majority of cases, the OC is both conceptually and empirically superior. Their account therefore does not preclude some sort of multi-level selection for culture: just as in the case of biological evolution, where there can be higher-level properties of groups that are not contained in any individual-sex ratio, for example-there can also be cultural traits that have not been adopted by any individual, but that have nevertheless been adopted by a group. For example, mask-making as a craft can be adopted by a group of individuals while none of the individuals of the group masters the craft as a whole. Organismic selection is the primary engine of adaptive change in evolution. But there are cases in which going up a level seems necessary, especially when there are new properties that exist only at the group level. (For Darwin in his (1871) Descent of Man, the proportion of altruists was just such a property.) Other cases are best understood as operating at the sub-organismic, genic level. Meiotic drive is one such example-this is a case in which particular alleles cheat the meiotic lottery and get their copies into the next generation at a disproportionately high frequency. When situations like this occur in cultural evolution, it is prudent to resort to a meme-centered approach. But, just as such situations are not the dominant evolutionary force in organisms, so, they suspect, is meme selection not the dominant force in human cultural evolution (though this is, ultimately, an empirical matter). If this is true, then, for the vast majority of cases, the Organism will be the best approach to cultural evolution.

Michael Vlerick's paper ("Explaining Universal Social Institutions: A Game-Theoretic Approach") addresses a question that remained largely neglected within classical social ontology (Searle 2010; Tuomela 2007)_-if it is true that large parts of our institutions depend, for their existence, on their collective acceptance by social groups, how is it that certain institutions (in particular, marriage, property and the regulation of common resources) seem to exist cross-culturally? This question, Vlerick contends, represents a challenge also for those theorists who, in contrast to social ontologists, trace back the existence of institutions to our mental wiring (Sperber 1996 and, more recently, Boyer and Petersen 2012): these latter accounts (while correct in highlighting the link between institutions and our mental dispositions) are unable to explain why certain institutions are universal or rather why similar institutions have emerged independently in different cultural contexts.
Vlerick's suggestion is the following: by relying on work done by Bowles and Gintis (Bowles and Gintis 2011), the author accepts the idea that one possibility to explain the spreading of altruistic behavior (which, again, is a necessary ingredient for the large-scale cooperation typical of human societies) is that cooperating groups have significant advantages vis à vis malfunctioning or non-cooperating groups - and that the high level of cooperation in the first groups is enabled by universal institutions. Put differently, universal institutions are "fitness peaks in the landscape of cultural possibilities," meaning that they secure altruism from free-riding behavior and, therefore, provide advantages at the group level. That is, a complete story of the emergence and development of institutions has to include their cultural dimension: institutions are (at least partly) products of cultural evolution.

Against this conceptual background, Vlerick models three fundamental institutions (property, common management and marriage) in game-theoretical terms by showing that institutions either modify the payoff matrix (insofar as, e.g., the institutions of property and common management introduce punishment for non-cooperative behavior) or create an additional motive to cooperate (in the case of marriage). And this fact, it is maintained, is the reason for their spreading through several cultures. This also leads the author to qualify institutions as 'gamechangers' for, once established, they change the rules and structure of the games and, hence, their outcome.

Institutions are also the topic of Jeppe Sinding Jensen's article ("How Institutions Work in Shared Intentionality and 'We-Mode' Social Cognition"), but the perspective with which institutions are approached here differs from the one adopted by Vlerick (although both lines of reasoning display important convergences, see below). Whereas Vlerick emphasizes the evolutionary dimension of institutions, Jensen's starting point is recent work in the theory of social cognition (especially Gallotti and Frith 2013) where he finds evidence for the idea that what social ontologists call the "we-mode" (cf. Tuomela 2007) has a neural correlate. The idea here is that the specific way of "having" an intentional attitude from a group perspective (one which also allows one to talk of thinking, intending or emoting in the "we-mode") is something grounded in human neural architecture.

However, it is contended that the introduction of this first level or basic kind of collective intentionality, legitimate as it is, is not yet sufficient to account for what Jensen calls "higher order shared intentionality." What is missed here are social institutions along with the normativity that accompanies them. On the one hand, institutions provide "cognitive governance" insofar as they act as stabilizers in social life, i.e., institutions seem to be able to reduce cognitive complexity, and they increase predictability in 
social interactions. On the other hand, they provide the interactants with roles, scripts and patterns that normatively shape their interaction.

Once institutions enter into the picture (and here a certain parallel with Vlerick's ideas seems to arise), social reality changes drastically, for institutions create "contexts" (i.e., normative programs for cognition, emotion and practice) in which the human mind just thinks differently. Jensen cashes out this idea by radicalizing Wilson's "Social Manifestation Thesis" (cf. Wilson 2004); in Jensen's words: "the social contexts are so 'loaded' by institutions that the groups function as if they had group minds." Said another way, the basic we-mode is seen as the first step towards establishing an institutional (and hence normative) reality, which again is a crucial step in activating fullblown collective intentionality. This makes human cognition predominantly cultural "in origins and in functions."

The idea that normativity is central for any account of social reality (and even of collective intentionality), already present in Jensen's paper, figures even more prominently in the article of Katharina Nieswandt, "Do Rights Exist by Convention or by Nature?"-where the focus is mainly put on moral normativity. The paper defends two interrelated claims: the first is that rights, all rights, exist by convention, and the second is that conventionalism about rights does not entail (cultural) relativism.

In her understanding of what a right is, Nieswandt follows Hohfeld (Hohfeld 1913): for a person to have a right is nothing but for others to have a duty towards that person to act in a particular way. According to the author, such duties can be justified in two different ways-either the justification solely appeals to the rules of a socially shared pattern of acting that impose the relevant duties, or the justification appeals to elements other than the rules of a socially shared pattern of acting. In the first case, we have a conventional right, in the second a right that, for the sake of labeling alone, Nieswandt calls "natural." (At this stage, it might be important to mention that, although perhaps irrelevant for the author's argument, the idea of "rules of a socially shared pattern of acting" closely resembles that of institution discussed in the two previous papers.)

Nieswandt's strategy is to show that, if this explanation of the notion of a right is sound, then all attempts to justify duties by recurring to the notion of a right necessarily lead to circularity. But, more importantly, the author also demonstrates that the attempts to justify duties (and, $a$ potiori, rights) by recurring to other facts, such as wellbeing, autonomy and divine commands, are all doomed to fail as they, too, run into circularity. The author does not exclude the possibility that there may be other "natural facts" that could come into consideration for the justification of duties (and, hence, her discussion simply establishes that "none of the currently available naturalist justifications of rights is plausible, not that no plausible justification could ever be found," our italics). And yet, apart from this caveat, her conclusion gives credence to the suggestion that there is no other way to justify duties than to ground them in shared patterns of acting.

But, then, how to square the conventionalist claim with a non-relativist view? The proposal, roughly, is to separate the account of rights and duties from the account of the relevant practices (and corresponding rules) that generate those duties (and hence those rights). Said another way, practices justify rights but, if the practices are not justified themselves, then the alleged rights do not have normative power and, thus, do not have to be respected. However, the justification of (at least) some practices must appeal to something that does not exist by convention. Therefore, although the author agrees that this non-relativist stance would have to be spelled out in further detail, it seems plausible that conventionalism about rights does not imply relativism about practices.

Corrado Roversi closes this issue with the introduction of the concept of institutional mimesis. According to the collective-intentionality view in social ontology, an institution exists if a set of rules of this kind is collectively accepted within a given community. But, for the most part, these rules are internalized by way of socialization, and this is where culture steps in: they are internalized within a given cultural framework. Culture cannot but play a crucial role in explaining why constitutive connections are framed in this way, rather than in another, or why they cannot be arbitrarily changed. In other words, while the structure of an institution is provided by constitutive rules, the rationale behind those constitutive rules is provided by assumptions that are at least in part culturally transmitted, and these assumptions can be tacit and are always the outcome of a specific historical development. Roversi makes his case by referring to a wide range of historical studies on the origins of hierarchies (Dubreuil 2010), the origins of the modern conception of the state (Toulmin 1990), and the origins of contracts (Hagerström 1941/1964).

\section{References}

Bowles S, Gintis H (2011) A cooperative species: human reciprocity and its evolution. Princeton University Press, Princeton

Boyer P, Petersen MB (2012) The Naturalness of (many) social institutions: evolved Cognition as their foundation. J Inst Econ $8(01): 1-25$

Dubreuil B (2010) Human evolution and the origins of hierarchies: the state of nature. Cambridge University Press, Cambridge

Gallotti M, Frith CD (2013) Social cognition in the we-mode. Trends Cogn Sci 17(4):160-165

Hagerström A (1941/1964) Philosophy and religion. Routledge, London

Hohfeld WN (1913) Some fundamental legal conceptions as applied in judicial reasoning. Yale Law J 23(1):16-59 
Searle J (2010) Making the social world: the structure of human civilization. OUP, Oxford

Skyrms B (2010) Signaling. OUP, Oxford

Sperber D (1996) Explaining culture: a naturalistic approach. Wiley, Oxford

Tomasello M (2008) The origins of human communication. MIT Press, Cambridge
Toulmin S (1990) Cosmopolis: the hidden agenda of modernity. University of Chicago Press, Chicago

Tuomela R (2007) The philosophy of sociality: the shared point of view. OUP, USA

Wilson RA (2004) Boundaries of the mind: the individual in the fragile sciences. Cambridge University Press, Cambridge 